\title{
Diffraction-managed superlensing using metallodielectric heterostructures
}

\author{
Carlos J. Zapata-Rodríguez, ${ }^{a}$ David Pastor, ${ }^{a}$ María T. Caballero, ${ }^{b}$ and Juan J. Miret ${ }^{b}$ \\ ${ }^{b}$ Department of Optics, University of Valencia, Dr. Moliner 50, 46100 Burjassot, Spain; \\ ${ }^{a}$ Department of Optics, Pharmacology and Anatomy, University of Alicante, P.O. Box 99, \\ Alicante, Spain
}

\begin{abstract}
We show that subwavelength diffracted wave fields may be managed inside multilayered plasmonic devices to achieve ultra-resolving lensing. For that purpose we first transform both homogeneous waves and a broad band of evanescent waves into propagating Bloch modes by means of a metal/dielectric (MD) superlattice. Beam spreading is subsequently compensated by means of negative refraction in a plasmon-induced anisotropic effectivemedium that is cemented behind. A precise design of the superlens doublet may lead to nearly aberration-free images with subwavelength resolution in spite of using optical paths longer than a wavelength.
\end{abstract}

Keywords: plasmonic devices, imaging, super-resolution

\section{INTRODUCTION}

Plasmonics is gaining current space in groundbreaking photonic technologies since it carries the possibility of moulding the flow of subwavelength wave fields. For instance, generation of nanosized hot spots by means of metallic nanoparticles and extraordinary transmission of thin films with subwavelength holes and slits have been extensively analyzed and observed in experiments. ${ }^{1,2}$ Relevant applications are high-density waveguiding, nanoantennas, labels for biomedical research and sensing to mention a few. ${ }^{3}$ In particular, superlenses are plasmonic flat devices capable of reconstruct a given scattered wave field with subwavelength features. ${ }^{4}$ This phenomenon has been applied successfully in high-resolution optical lithography. ${ }^{5,6}$

The image formation by metallic single-layer superlenses is based on the excitation of surface plasmons polaritons (SPPs) and anti-plasmons on the entrance and exit interfaces. ${ }^{7}$ As a consequence wave amplification inside the metallic slab compensates the attenuation produced in the surrounding dielectric. By coupling a few of these elementary thin lenses we may compose a MD multilayered device capable of transmit high-frequency plane waves by resonant tunneling. ${ }^{8,9}$ Assuming a perfect periodic process, a set of evanescent waves are effectively converted into propagating Bloch waves with characteristic pseudo-moment and consequently carrying electromagnetic energy.

Two main concerns are critical in order to achieve a high-fidelity replica of the scattered field. First we will prevent resonant peaks in the transmittance response that would enhance certain spatial frequencies and thus disfiguring the image. Secondly we shall preclude dephasing of different spectral components that appear in the transmission coefficient. The first issue may be treated essentially by using metals and dielectrics whose permittivities are selectively adjusted, and the second subject is addressed by tuning the filling factors of the materials involved. As a result the wave fields will propagate inside the multilayered metallic composites in the canalization regime. ${ }^{10,11}$

In this contribution we follow a different approach that leads to control dephasing of subwavelength outputs. This is based on counterbalancing the phase response of high-transparent metallic superlenses in order to flatten the overall phase mismatch at the image plane. In geometrical terms, negative refraction of high-frequency Bloch waves excited by a nanosized object contributes to achieve a nearly-stigmatic subwavelength image. Our investigation is inspired on the direct observation of light focusing through a photonic crystal flat lens designed

Further author information: (Send correspondence to C.J.Z.R.)

C.J.Z.R.: E-mail: carlos.zapata@uv.es, Telephone: +34963543805

Metamaterials VII, edited by Allan D. Boardman, Nigel P. Johnson, Richard W. Ziolkowski, Proc. of SPIE Vol. 8423, 84230U · ( 2012 SPIE · CCC code: 0277-786X/12/\$18 · doi: 10.1117/12.921882 


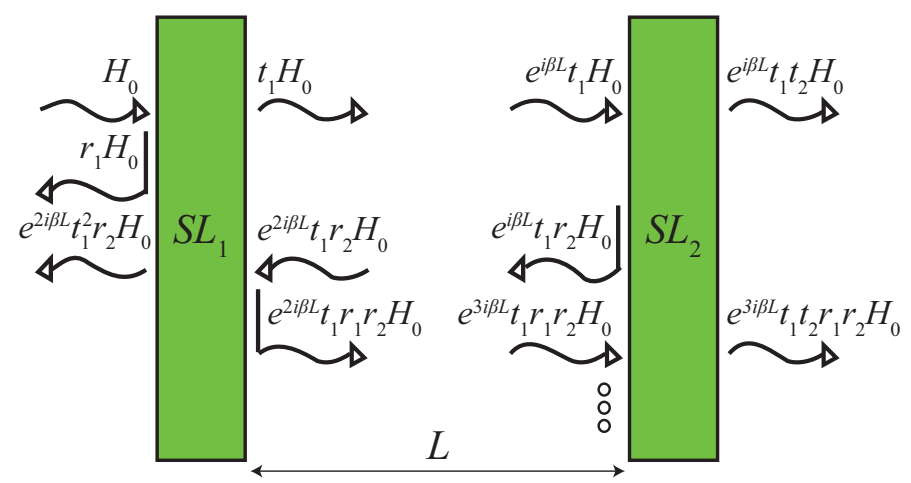

Figure 1. Illustration of the generalized Airy's formula for two coupled superlenses, $S L_{1}$ and $S L_{2}$.

and fabricated using a III-V semiconductor slab to operate at optical frequencies. ${ }^{12}$ More sophisticated devices following the above primitive idea has been presented recently. ${ }^{13}$ However the spot size of the image field is clearly diffraction limited. A recent tentative to overcome this limit by using uniform arrays of plasmonic waveguides has been proposed elsewhere. ${ }^{14,15}$ Here we discuss the potential advantages of our proposal based on the coupling of MD superlenses. Part of this study has been reported elsewhere. ${ }^{16}$

\section{THE PHASE ADDITION RULE}

In this section we examine the role of elementary flat superlenses in the optical response of a two-fold coupled plasmonic device. For that purpose let us consider two thin superlenses that, for simplicity, are immersed in the same transparent medium of dielectric constant $\epsilon$. In principle our approach holds for superlenses that are formed by a single metallic layer, but here we are focused on multilayered structures. When a given TM plane wave of amplitude $H_{0}$ and in-plane spatial frequency $k_{x}$ impinges on the front face of the first superlens, the wave field is partially transmitted with a complex amplitude $t_{1} H_{0}$; therefore $t_{1}$ stands for the coefficient of transmission. In the case that absorption and reflections are negligible, $\left|t_{1}\right| \approx 1$ for homogeneous waves in the host medium but this is not necessarily true for evanescent fields, where $\left|k_{x}\right|>\sqrt{\epsilon} k_{0}$ and $k_{0}=2 \pi / \lambda_{0}$ is the wavenumber in vacuum. Propagation along the intermediate medium results in an additional factor $\exp (i \beta L)$, where $\beta=\sqrt{\epsilon k_{0}^{2}-k_{x}^{2}}$. The following step consists of traversing thought the second metallic near-field flat lens. Assuming a coefficient of transmission $t_{2}$ for the second superlens, the transmitted field yields $t_{1} t_{2} \exp (i \beta L) H_{0}$. More generally, the Airy's formula ${ }^{17}$ would take into account multiple scattered waves giving a transmitted field

$$
H_{y}=t_{1} t_{2} \exp (i \beta L) H_{0}\left[1+r_{1} r_{2} \exp (2 i \beta L)+r_{1}^{2} r_{2}^{2} \exp (4 i \beta L)+\ldots\right]=\frac{t_{1} t_{2} \exp (i \beta L) H_{0}}{1-r_{1} r_{2} \exp (2 i \beta L)},
$$

where $r_{1,2}$ is the coefficient of reflection corresponding to a given superlens. This is illustrated in Fig. 1.

In our discussion we will consider that $S L_{1}$ and $S L_{2}$ are firmly attached and, as a consequence, we set $L=0$ from here on. From Eq. (1) we infer that the phase increment of the transmitted wave with respect to the incident field is the result of a summation of three terms, corresponding to the arguments of the phasors $t_{1}, t_{2}$, and $\left(1-r_{1} r_{2}\right)^{-1}$, respectively. In this paper this is called the phase addition rule. In the case that the modulus of either $r_{1}$ or $r_{2}$ is significantly lower than unity, the last phasor may be neglected and the phase of the emerging field is directly controlled by the arguments of the transmission coefficients $t_{1,2}$. In this sense, the dependence of $\arg \left(t_{1}\right)$ upon $k_{x}$ might be compensated with a prescribed superlens $S L_{2}$ leading to a ultraflattened curve at least within a given spectral band. Otherwise the argument of $\left(1-r_{1} r_{2}\right)^{-1}$ may transform the overall phase variation in a nonlinear way. Next we consider a procedure to play on the phase addition rule in order to achieve subwavelength aberration-free images.

\section{WAVE ABERRATION COMPENSATION}

In Fig. 2 we represent the spectral dependence of the transmission coefficient both in amplitude and phase for a couple of metallodielectric multilayered devices at $\lambda_{0}=485 \mathrm{~nm}$. Fig. 2(a) shows the transmission coefficient 

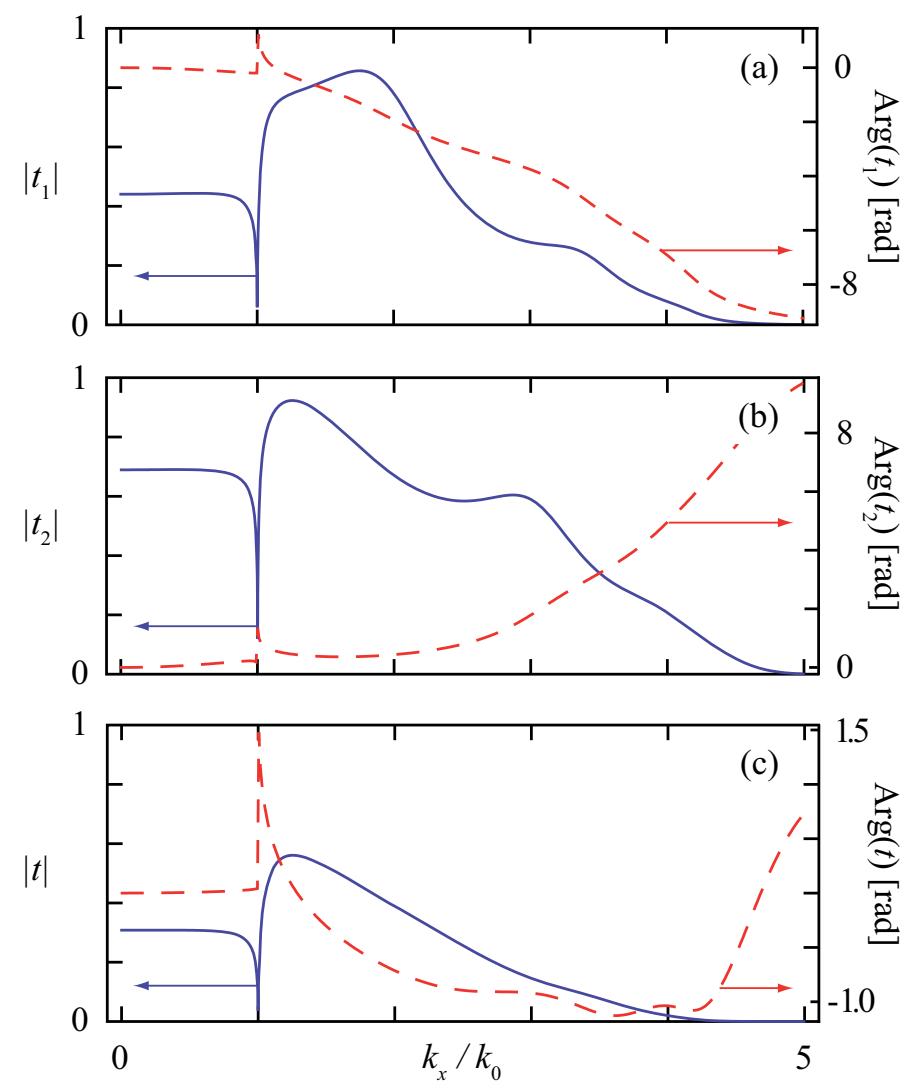

Figure 2. Transmission coefficient in modulus and phase for finite periodic structures made of silver and a dielectric: (a) $\mathrm{SrTiO}_{3}$; (b) GaP. In (a) we have $N_{1}=19$ silver layers with a filling factor of $f_{1}=0.35$ within a period of $\Lambda_{1}=30 \mathrm{~nm}$. In (b) we consider $N_{2}=7$ periods of $\Lambda_{2}=50 \mathrm{~nm}$ for a silver filling factor $f_{2}=0.50$. In both stacked devices the surrounding medium is the vacuum. (c) The transmission coefficient of the coupled structure.

for a periodic structure consisting of $N_{1}=20$ silver layers $\left(\epsilon_{\mathrm{Ag}}=-7.754+i 0.727\right)$ hosted in $\mathrm{SrTiO}_{3}\left(\epsilon_{\mathrm{SrTiO}_{3}}=\right.$ $6.596+i 0.070)$. The unit cell has a period of $\Lambda_{1}=30 \mathrm{~nm}$, and the filling factor of silver is $f_{1}=0.35$. Also the surrounding medium of the stack is considered to be the vacuo. From the figure of $\left|t_{1}\right|$ we observe that, in practical terms, our finite lattice cannot transmit spatial frequencies beyond $k_{x}=\kappa k_{0}$, where the effective index of refraction $\kappa=4.5$ in our numerical example. Since the refractive index of $\mathrm{SrTiO}_{3}$, that is $n_{\mathrm{SrTiO}_{3}}=\operatorname{Re} \sqrt{\epsilon_{\mathrm{SrTiO}_{3}}}=2.57$, is considerably lower than $\kappa=4.5$, a resonant tunneling effect driven by SPPs is evident. Moreover, a decreasing variation of the phase of $t_{1}$ in terms of $k_{x}$ is clearly revealed. In Fig. 2(b) we depict the transmission coefficient for a second lattice made of $N_{2}=7$ silver slabs placed on $\mathrm{GaP}\left(\epsilon_{\mathrm{GaP}}=13.287\right)$. In this case the period is $\Lambda_{2}=50 \mathrm{~nm}$ and the filling factor of silver is $f_{2}=0.50$. While the effective index of refraction $\kappa$ is comparable with that obtained in the previous case, the phase shows a completely different behaviour. Now the complex argument of the transmittance increases for higher spatial frequencies $k_{x}$. Note also that the phase increment observed in the GaP lattice goes around $3 \pi$ rads, which approximately corresponds to the phase decrement attributed to the $\mathrm{SrTiO}_{3}$ multilayer. Then a coupled device including both types of periodic nano-structures would yield a compensated-phase response. This finding is confirmed in Fig. 2(c) where the net phase deviation is always lower than $\pi$ rads within the effective bandwidth $\left|k_{x}\right|<5 k_{0}$.

The different behavior observed in the phase dependence of the transmittance upon spatial frequencies may be explained from the isofrequency curves of the periodic lattices. In Fig. 3 we represent the dispersion equation ${ }^{17}$

$$
\cos \left(k_{z} \Lambda\right)=\cos \varphi_{1} \cos \varphi_{2}-\eta \sin \varphi_{1} \sin \varphi_{2},
$$

corresponding to P-polarized waves propagating within an infinite periodic multilayered structure, where the 
(a)

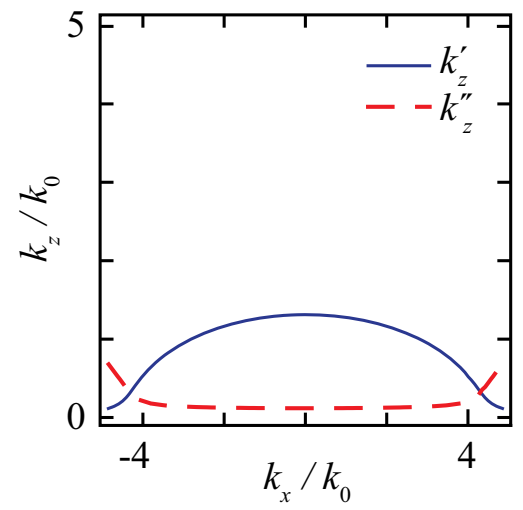

(b)

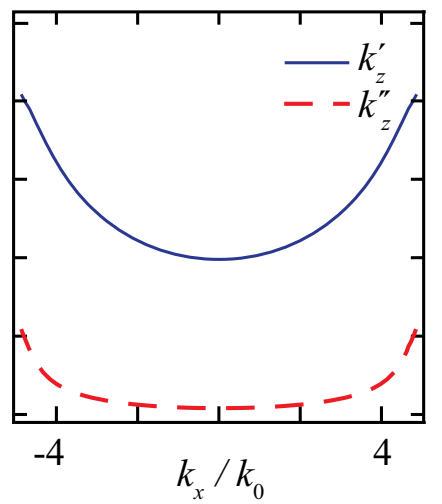

Figure 3. Isofrequency curves given from Eq. (2) for the hybrid silver-dielectric periodic media containing (a) $\mathrm{SrTiO}_{3}$ and (b) GaP. Note that $k_{z}^{\prime}$ and $k_{z}^{\prime \prime}$ are the real part and the imaginary part of $k_{z}$, respectively.

metal-dielectric interfaces are parallel to the $x y$ plane. Therefore the component $k_{z}=k_{z}^{\prime}+i k_{z}^{\prime \prime}$ of the wave vector represents a Bloch pseudo-moment. In Eq. (2) the period of the structure is $\Lambda=d_{1}+d_{2}$, also $\varphi_{q}=k_{q z} d_{q}$ with $q=\{1,2\}$ representing either the dielectric or the metal, $2 \eta=\epsilon_{1} k_{2 z} / \epsilon_{2} k_{1 z}+\epsilon_{2} k_{1 z} / \epsilon_{1} k_{2 z}$, and finally assuming $k_{y}=0$ then $k_{q z}^{2}+k_{x}^{2}=\epsilon_{q} k_{0}^{2}$ represents the dispersion equation within each bulk medium. From Fig. 3 we infer that the superlattice made of $\mathrm{SrTiO}_{3}$ has an isofrequency curve with normal negative curvature. The propagator $\exp \left(i k_{z}^{\prime} z\right)$ indicates that the dephase accumulated by a wave field is directly proportional to $k_{z}^{\prime}$. Neglecting impedance mismatch at the input and output planes of the multilayered device, we expect that the dependence of $k_{z}^{\prime}$ upon $k_{x}$ follows the same variation observed upon the transmission coefficient. Figures 2(a) and 3(a) indicate that this is in good agreement up to the normalized cutoff frequency $\kappa$ given by the solution of the equation $k_{z}^{\prime}\left(\kappa k_{0}\right)=k_{z}^{\prime \prime}\left(\kappa k_{0}\right)$ that is $\kappa=4.3$. Now we may follow a similar procedure to relate the isofrequency curve given in Fig. 3(b), which corresponds to the periodic medium containing GaP, with the phase spectral dependence of the transmission coefficient given in Fig. 2(b). In this case we conclude that the positive curvature of the dispersion equation explains the phase increment observed at higher spatial frequencies $k_{x}$.

Phase compensation attributed to coupling of two MD superlattices with isofrequencies of opposite curvature also renders a geometrical interpretation of our results. For the sake of clarity let us consider the propagation direction of a monochromatic wave packet with carrier spatial frequency $k_{x 0}$. This wave field flies within a periodic medium along a direction given by the normal vector $\vec{N}=\left(N_{x}, N_{z}\right)$, which is calculated from the isofrequency curve in terms of the group velocity $\vec{v}_{g}=\nabla_{\vec{k}} \omega$ in the vicinities of $k_{x 0}$ and $\omega_{0}$. Assuming that the field propagates in the positive direction of the $z$ axis, that is $N_{z}>0$, we find that the sign of $N_{x}$ depends exclusively on the curvature of the isofrequency curve. As a consequence, the wave packet that pass through the interface of both MD superlattices experiences negative refraction. This is illustrated in Fig. 4 for a wave packet of carrier frequency (a) $k_{x 0}=k_{0}$ and (b) $k_{x 0}=3 k_{0}$. The numerical simulations are performed with a commercial software based on the finite-element method (FEM). Since attenuation driven by losses in silver makes difficult a clear observation of the wave-packet trajectory, also we depict the transverse magnetic field normalized in such a way that the maximum value of $\left|H_{y}\right|$ at a given plane $z=z_{0}$ is always the unity. When the fields propagate within the lattice composed of $\mathrm{SrTiO}_{3}$, the angles corresponding with each vector $\vec{N}$ as measured with respect to the $z$ axis are numerically estimated from Figs. 3(a) and (b) as $\theta_{1}=+0.07$ rads and $\theta_{1}=+0.28 \mathrm{rads}$, respectively. Note that these angles are significantly small. If the wave packets travel through the multilayered medium containing GaP, these angles yield $\theta_{2}=-0.12$ rads and $\theta_{2}=-0.44$ rads. We point out that in both cases $C=\tan \theta_{1} / \tan \theta_{2} \approx-0.62$; moreover $C$ is a quantity that is approximately conserved for $\left|k_{x}\right|<3.5 k_{0}$. As a consequence a light ray emerging from a point on axis that propagates from a plane $z=0$ to $z=L_{1}$ in the first medium and, immediately after, travels in the second medium up to $z=L_{1}+L_{2}$, such that $C=-L_{2} / L_{1}$ holds, gets its way back to the $z$ axis.

In principle, this property holds for a wide spectral range. This is important if we consider a localized 
(a)

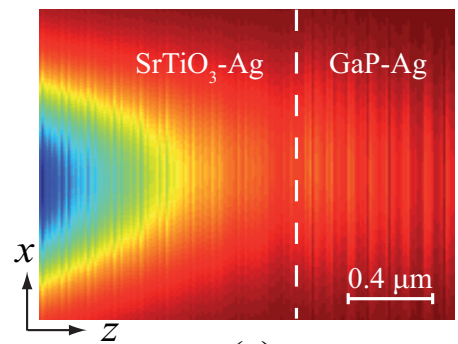

(c)

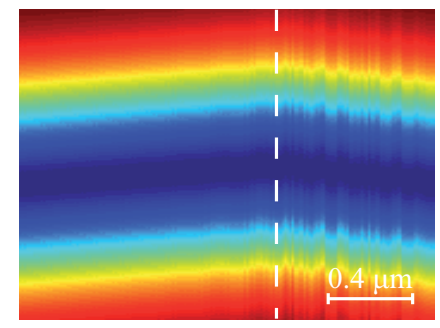

(b)

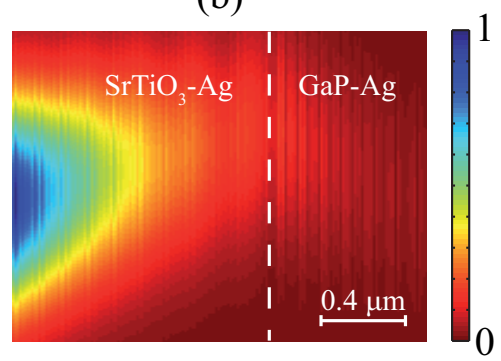

(d)

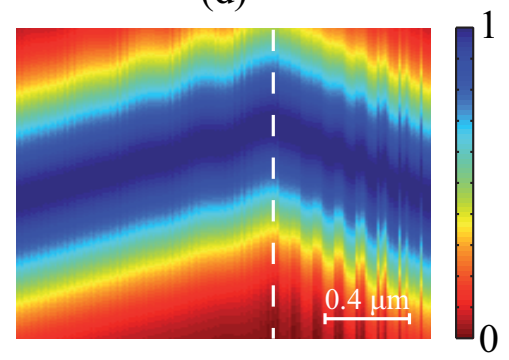

Figure 4. FEM simulations shown negative refraction on the surface (black dashed line) that joins the MD superlattice including $\mathrm{SrTiO}_{3}$, set on the left, and that containing GaP. Sub-figures (c) and (d) result from normalizing the modulus of the magnetic field $\left|H_{y}\right|$ plane by plane.

(a)

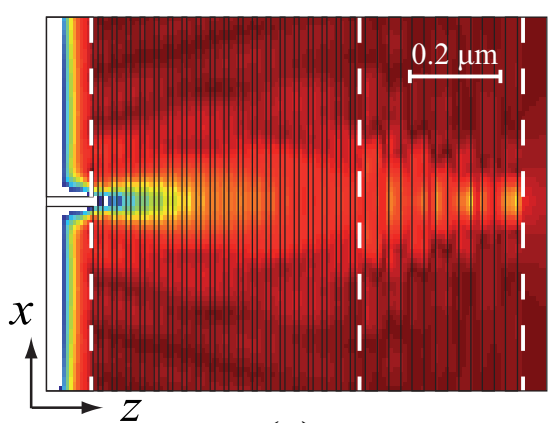

(c)

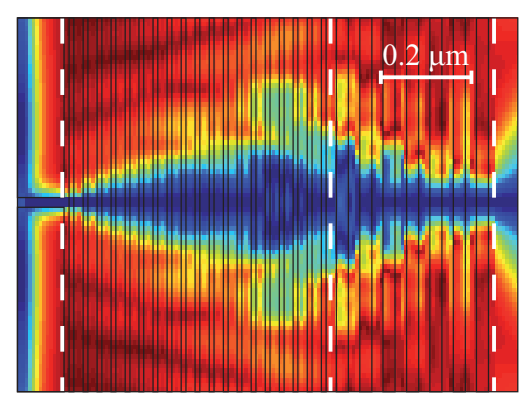

(b)

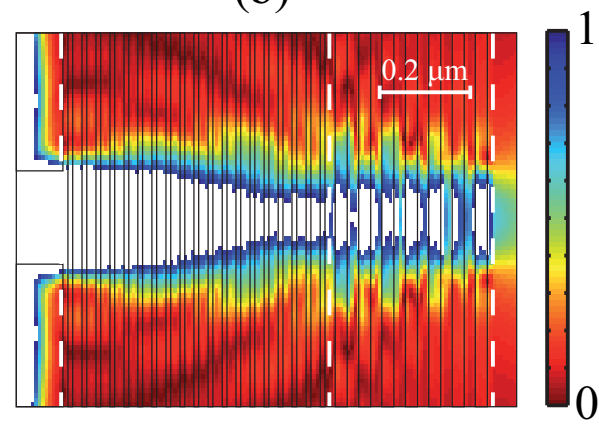

(d)

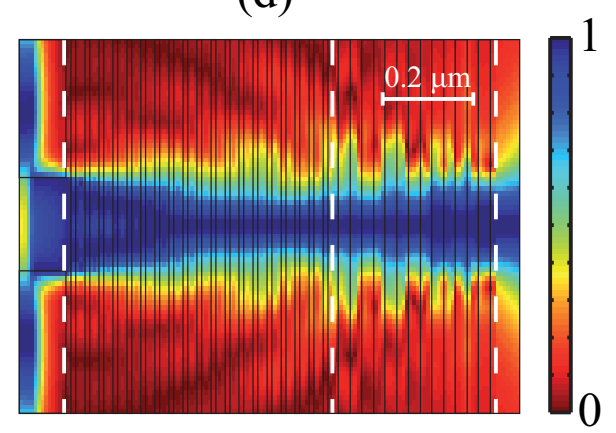

Figure 5. FEM simulations shown superlensing of the device composing the MD superlattice including $\mathrm{SrTiO}_{3}$, set on the left, and that containing GaP. In (a) the slit width is $20 \mathrm{~nm}$ and in (b) the slit width is $200 \mathrm{~nm}$. For the sake of clarity, again we show the modulus of the magnetic field in (c) and (d) as it is normalized plane by plane. 


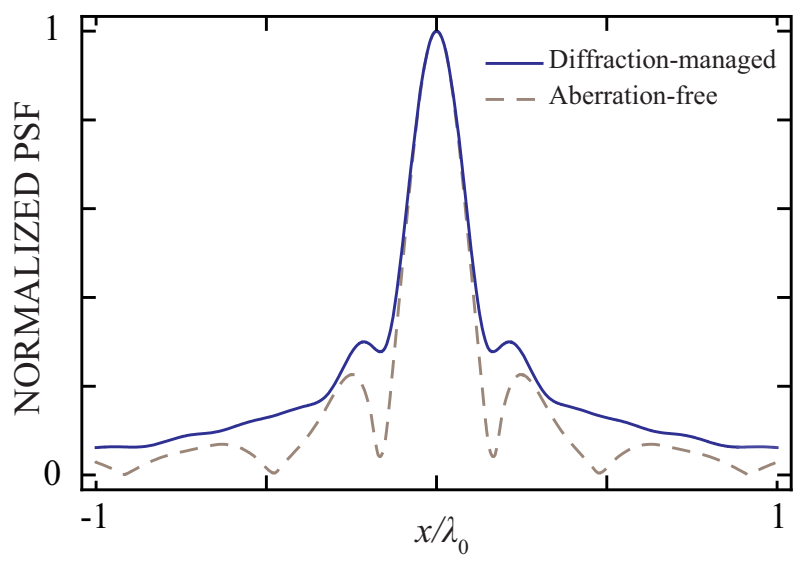

Figure 6. Modulus of the 1D PSF at the image plane of our diffraction-managed superlens. The 1D PSF of an aberrationfree system maintaining the same $|t|$ is also represented graphically.

source in the input interface of the first MD finite lattice. The light rays emerging from the point object are conveniently deviated at the surface that separates the periodic media, by means of negative refraction, in such a way that at the plane $z=L_{1}+L_{2}$ all of them are focused. In order words, the condition of stigmatism is approximately satisfied so that nearly aberration-free images may be formed by our device. We point out that spherical aberration is not completely removed in this study, a fact that would decrease the resolving power of the imaging system. ${ }^{18}$ This is illustrated in Fig. 5 by using FEM simulations in COMSOL Multiphysics. In front of our device we insert a $\mathrm{Cr}$ layer of width $100 \mathrm{~nm}$ with a centered slit aperture of $20 \mathrm{~nm}$-width in Fig. 5(a). A P-polarized plane wave is incident onto the Cr film that collects part of the light, which is guided toward the entrance surface of the diffraction-managed superlens. Thus the deep-subwavelength wave field in the input is diffracted inside the first multilayered medium; subsequently it is compressed in the transverse direction as it propagates along the GaP superlattice. The output magnetic field consists of a strong central lobe whose FWHM is $\rho=130 \mathrm{~nm}$, that represents only $0.27 \lambda_{0}$. This confirms the subwavelength character of the image-formation process in spite of the fact that the object plane and the image plane are separated by a distance of $950 \mathrm{~nm}$ that supposes barely twice the wavelength. We have repeated the FEM simulations for other slit widths, and we have observed that the response of the superlensing device is practically the same whether the slit width is substantially smaller that $\rho$. Therefore $\rho$ stands for the limit of resolution of the MD superlens. For wider slits, on the contrary, the magnetic field at the output plane resembles that at the input plane. In Fig. 5(b) we show the wave field for a slit aperture of $200 \mathrm{~nm}$. In this case, the beam width that is excited by the plasmonic slit is conserved not only at the exit but all along the periodic media. This canalization regime is the result of the strong anisotropy of the two superlattices involved, as shown in Fig. 3. Furthermore, the low-frequency of the spatial spectrum associated with the scattered electromagnetic waves at the input plane, in this case, leads to the dominant canalization of the fields.

To estimate the limit of resolution we alternatively employ the so-called 1D point spread function (PSF). For a multilayered device, the 1D PSF is simply the 1D Fourier transform of the transmission coefficient $t\left(k_{x}\right)$ for TM polarization. Obviously, $t\left(k_{x}\right)$ is the ratio of the magnetic field at the output plane and that at input plane, respectively, provided a P-polarized plane wave of transverse spatial frequency $k_{x}$. Strictly speaking this 1D PSF is valid for line sources instead of point sources, the latter case treated elsewhere. ${ }^{19,20}$ In mathematical terms we write ${ }^{21}$

$$
h(x)=\frac{1}{2 \pi} \int_{-\infty}^{\infty} t\left(k_{x}\right) \exp \left(i k_{x} x\right) d k_{x} .
$$

From a practical point of view, the coefficient $t$ is simply estimated by using a transfer $2 \times 2$ matrix formulation. ${ }^{17}$ Fig. 6 depicts the modulus of 1D PSF at the image plane of our diffraction-managed device. The FWHM of the central peak shown by $|h(x)|$ is $0.214 \lambda_{0}$, which is very close to the value of $\rho$ obtained from FEM-based numerical simulations. For the sake of completeness we also represented the 1D PSF for a purely aberration-free 
setup with the same transmission strength $\left|t\left(k_{x}\right)\right|$. After performing the corresponding 1D Fourier transform of $\left|t\left(k_{x}\right)\right|$ we estimate that the FWHM of the diffraction-free 1D PSF decreases up to a value of $0.195 \lambda_{0}$. We conclude that the 1D impulse response is not blurred significantly due to aberrations. This is also confirmed by simply inspection of the small strength associated with the closest sidelobes of the 1D PSF, a fact that is of relevance in the image formation of either localized scatterers or extended objects.

\section{CONCLUSIONS}

We conclude that management of subwavelength diffracted wave fields may be employed in multilayered flat lenses to obtain superresolution. A first MD superlattice with strong anisotropy converts evanescent fields into propagating Bloch modes. Beam spreading is compensated by using negative refraction. For that purpose, a second MD superlattice with an isofrequency curve of opposite curvature collects the wide spectrum of incoming Bloch waves and, after that, they are suitably focused just at the output plane of the superlensing device. Although our results are highly satisfactory, we believe that substantial improvements may be considered in future studies.

\section{ACKNOWLEDGMENTS}

This research was funded by the Spanish Ministry of Economy and Competitiveness under the project TEC200911635 .

\section{REFERENCES}

1. T. Klar, M. Perner, S. Grosse, G. von Plessen, W. Spirkl, and J. Feldmann, "Surface-plasmon resonances in single metallic nanoparticles," Phys. Rev. Lett. 80, pp. 4249-4252, 1998.

2. L. Martín-Moreno, F. J. García-Vidal, H. J. Lezec, K. M. Pellerin, T. Thio, J. B. Pendry, and T. Ebbesen, "Theory of extraordinary optical transmission through subwavelength hole arrays," Phys. Rev. Lett. 86, pp. 1114-1117, 2001.

3. M. I. Stockman, "Nanoplasmonics: past, present, and glimpse into future," Opt. Express 19, pp. 22029 22106, 2011.

4. J. B. Pendry, "Negative refraction makes a perfect lens," Phys. Rev. Lett. 85, pp. 3966-3969, 2000.

5. N. Fang, H. S. Lee, C. Sun, and X. Zhang, "Sub-diffraction-limited optical imaging with a silver superlens," Science 308, pp. 534-537, 2005.

6. D. O. S. Melville and R. J. Blaikie, "Super-resolution imaging through a planar silver layer," Opt. Express 13, pp. 2127-2134, 2005.

7. J. B. Pendry and S. A. Ramakrishna, "Refining the perfect lens," Physica B 338, pp. 329-332, 2003.

8. M. Scalora, M. J. Bloemer, A. S. Pethel, J. P. Dowling, C. M. Bowden, and A. S. Manka, "Transparent, metallo-dielectric, one-dimensional, photonic band-gap structures," J. Appl. Phys. 83, pp. 2377-2383, 1998.

9. E. Shamonina, V. A. Kalinin, K. H. Ringhofer, and L. Solymar, "Imaging, compression and Poynting vector streamlines with negative permittivity materials," Electron. Lett. 37, pp. 1243-1244, 2001.

10. P. A. Belov and Y. Hao, "Subwavelength imaging at optical frequencies using a transmission device formed by a periodic layered metal-dielectric structure operating in the canalization regime," Phys. Rev. B 73, p. $113110,2006$.

11. A. Pastuszczak and R. Kotyński, "Optimized low-loss multilayers for imaging with sub-wavelength resolution in the visible wavelength range," J. Appl. Phys. 109, p. 084302, 2011.

12. N. Fabre, L. Lalouat, B. Cluzel, X. Mélique, D. Lippens, F. de Fornel, and O. Vanbésien, "Optical near-field microscopy of light focusing through a photonic crystal flat lens," Phys. Rev. Lett. 101, p. 073901, 2008.

13. S. Kocaman, M. S. Aras, P. Hsieh, J. F. McMillan, C. G. Biris, N. C. Panoiu, M. B. Yu, L. Kwong, A. Stein, and C. W. Wong, "Zero phase delay in negative-refractive-index photonic crystal superlattices," Nature Photonics 5, pp. 499-505, 2011.

14. M. Conforti, M. Guasoni, and C. D. Angelis, "Subwavelength diffraction management," Opt. Lett. 33, pp. 2662-2664, 2008. 
15. C. Yan, D. H. Zhang, Y. Zhang, D. Li, and M. A. Fiddy, "Metal-dielectric composites for beam splitting and far-field deep sub-wavelength resolution for visible wavelengths," Opt. Express 18, pp. 14794-14801, 2010.

16. C. J. Zapata-Rodríguez, D. Pastor, M. T. Caballero, and J. J. Miret, "Diffraction-managed superlensing using plasmonic lattices," Opt. Commun., submitted.

17. P. Yeh, Optical Waves in Layered Media, Wiley, New York, 1988.

18. C. J. Zapata-Rodríguez, D. Pastor, and J. J. Miret, "Metamaterial coatings for subwavelength-resolution imaging," in Proc. SPIE Vol. 8070, V. Kuzmiak, P. Markos, and T. Szoplik, eds., p. 807041, 2011.

19. C. J. Zapata-Rodríguez, D. Pastor, and J. J. Miret, "Three-dimensional point spread function and generalized amplitude transfer function of near-field flat lenses," Appl. Opt. 49, pp. 5870-5877, 2010.

20. C. J. Zapata-Rodríguez, D. Pastor, V. Camps, M. T. Caballero, and J. J. Miret, "Three-dimensional point spread function of multilayered flat lenses and its application to extreme subwavelength resolution," $J$. Nanophoton. 5, p. 051807, 2011.

21. R. Kotyński, "Fourier optics approach to imaging with sub-wavelength resolution through metal-dielectric multilayers," Opto-Electron. Rev. 18, p. 366, 2010. 